\title{
Endurance exercise increases the protein levels of PGC-1a and respiratory chain complexes in mouse skeletal muscle during atorvastatin administration
}

\author{
Koji Nonaka ${ }^{1}$ (1) $\cdot$ Yutaka Ozaki $^{2} \cdot$ Kenichi Ito $^{3} \cdot$ Masahiro Sakita $^{1} \cdot$ Satsuki Une ${ }^{4} \cdot$ Junichi Akiyama $^{5}$
}

Received: 28 June 2018 / Accepted: 27 November 2018 / Published online: 8 December 2018

(C) The Physiological Society of Japan and Springer Japan KK, part of Springer Nature 2018

\begin{abstract}
Statins and exercise reduce cardiovascular disease incidence. We investigated whether endurance exercise in mice induces mitochondrial adaptation in skeletal muscle and muscle injury during administration of atorvastatin, a member of the statin medication class. Male C57BL mice were assigned to one of three groups: control (Con), statin (Statin), or statin and exercise $($ Statin + Ex). Atorvastatin was administered, and exercise performed on a treadmill for 8 weeks. The levels of mitochondriaassociated proteins, PGC- $1 \alpha$, and respiratory chain complex, (COX) I-V, in the quadriceps femoris, and serum creatine kinase, a muscle injury marker, were measured. PGC-1 $\alpha$ and COX I-V were upregulated in the Statin + Ex group compared to those in the Statin and Con groups; serum creatine kinase levels were similar. Endurance training in mice induced mitochondrial adaptation in skeletal muscle without causing muscle injury, during atorvastatin administration.
\end{abstract}

Keywords Statin $\cdot$ Exercise $\cdot$ PGC-1 $1 \alpha \cdot$ Respiratory chain complexes $\cdot$ Muscle injury

\section{Introduction}

Statins [3-hydroxy-3-methyl-glutaryl coenzyme A reductase (HMG-CoA reductase) inhibitors] are cholesterol-lowering drugs [1] and have been reported to prevent cardiovascular mortality and events [2]. Exercise has also been reported to reduce cardiovascular disease incidence [3]. Therefore, it would seem advisable for statin users to also engage in exercise. However, the effects of exercise on statin users are not clearly understood. In addition, exercise is suggested

Koji Nonaka

nonaka-k@tachibana-u.ac.jp

1 Department of Physical Therapy, Faculty of Health Sciences, Kyoto Tachibana University, 34 Oyakeyamada-cho, Yamashina-ku, Kyoto, Kyoto 607-8175, Japan

2 Department of Rehabilitation, Kansai Electric Power Hospital, Osaka, Japan

3 Faculty of Health Sciences, Naragakuen University, Nara, Japan

4 Faculty of Education, Kagawa University, Takamatsu, Kagawa, Japan

5 Department of Physical Therapy, School of Health Care and Social Welfare, Kibi International University, Takahashi, Okayama, Japan to exacerbate muscle injury during statin administration. Hence, the safety of daily exercise for statin users is unclear.

Endurance exercise is well known to stimulate metabolic adaptation, such as enhancement of oxidative phosphorylation in skeletal muscle [4]. However, it is not clear whether exercise has adaptive effects during statin administration because statins can potentially induce mitochondrial dysfunction [5]. Southern et al. have reported that exercise increases activities of mitochondrial enzymes such as citrate synthase and $\beta$-hydroxy acyl-CoA dehydrogenase in the skeletal muscle of mice administered statins [6], and they indicated that exercise induces mitochondrial adaptation during statin administration. In contrast, Mikus et al. demonstrated that statins impair exercise-induced metabolic adaptation in humans [7]. Thus, it is unclear whether exercise has adaptive effects on metabolic factors related to mitochondria during statin administration.

There are also possible safety concerns for statin users concerning the performance of daily exercise because exercise may result in muscle injury during statin administration. Exercise has been reported to exacerbate injury in the gastrocnemius, extensor digitorum longus, and psoas muscles of mice administered cerivastatin $(1 \mathrm{mg} / \mathrm{kg})$ [8]. However, in that study, the quadriceps femoris of all the mice that did not engage in exercise were reported to be damaged. Hence, 
exacerbation of muscle injury does not appear to be due to only exercise but also cerivastatin, which is not currently prescribed owing to its strong side effects. In another study, lovastatin increased levels of creatine kinase (CK), which is a marker of muscle injury, with architecture disruption and plasma membrane damage during downhill treadmill walking, which is known as eccentric exercise [9]. Eccentric exercise was shown to induce muscle injury, with necrotic fibers [10] accompanying muscle soreness [11]. Patients who are administered statins usually do not perform such injurious exercises as part of their daily exercise regimen. Moreover, exercise increases the expression of heat shock proteins (HSPs) [12], which may potentially protect muscle from injury [13]. Therefore, it is unclear whether exercise induces muscle injury during statin administration.

The purpose of this study was to investigate whether exercise exerts adaptive effects on mitochondria and induces muscle injury during statin administration. Levels of peroxisome proliferator-activated receptor gamma coactivator 1-alpha (PGC-1 $\alpha$ ) and respiratory chain complex (COX) $\mathrm{I}-\mathrm{V}$, which are mitochondrial-associated proteins, were measured to determine the resulting adaptive effects of exercise. Moreover, serum CK activity levels were measured and skeletal muscle tissues were observed by microscopy to verify muscle fiber disruption as an indication of muscle injury. In addition, HSP25 levels were measured because they are increased by endurance exercise and have a protective effect against stress.

\section{Methods}

\section{Animals}

Eighteen male C57BL mice (8 weeks old) were housed in a temperature-controlled room maintained at $22{ }^{\circ} \mathrm{C}$ under a 12-h light-dark cycle and allowed free access to food and water. The mice were randomly divided into three groups consisting of six animals per group as follows: control (Con), statin (Statin), and statin and exercise (Statin + Ex). The study was approved by the Institutional Animal Care and Use Committee of Osaka Prefecture University.

\section{Atorvastatin treatment}

Mice in the statin treatment groups were treated with a dose of $10 \mathrm{mg} / \mathrm{kg} /$ day of atorvastatin calcium salt trihydrate (Tokyo Chemical Industry Co., Ltd., Tokyo, Japan) for 8 weeks. The dose of atorvastatin calcium salt trihydrate was determined according to a previous study [14]. The suspension of atorvastatin was prepared in $0.5 \% \mathrm{w} / \mathrm{v}$ methyl cellulose 400 solution (Wako Pure Chemical Industries, Ltd., Osaka, Japan) and administered to the mice in the statin treatment groups orally using a feeding needle, daily for 8 weeks. The $0.5 \%$ w/v methyl cellulose 400 solution was given to the mice in the control group for 8 weeks.

\section{Exercise protocol}

The exercise protocol was determined based on a previous study [15]. The mice in the Statin + Ex group ran on a motordriven treadmill (Muromachi Kikai Co., Ltd., Tokyo, Japan) at $15 \mathrm{~m} / \mathrm{min}$ for $60 \mathrm{~min}$. The exercise was performed once a day, 5 days a week for 8 weeks.

\section{Serum and tissue sampling}

The mice were deeply anesthetized with isoflurane at $24 \mathrm{~h}$ after the last exercise treatment, and blood was collected from the heart. The quadriceps femoris muscles were collected. Blood was centrifuged at $1200 \times g$ for $10 \mathrm{~min}$ at $4{ }^{\circ} \mathrm{C}$, and then the serum was collected. The muscles were immediately frozen in isopentane cooled with liquid nitrogen, followed by storage at $-80{ }^{\circ} \mathrm{C}$ until further analysis.

\section{Biochemical analysis}

The serum level of total cholesterol, LDL cholesterol, and CK was measured with a 7180 automatic analyzer (Hitachi, Tokyo, Japan), using L-type Wako CHO M (Wako Pure Chemical Industries Ltd., Osaka, Japan), Cholestest LDL (Sekisui Medical CO., Ltd., Tokyo, Japan), and L-type Wako CK (Wako Pure Chemical Industries Ltd.).

\section{Western blotting}

The muscles were homogenized in ice-cold extraction buffer $(20 \mathrm{mM}$ Tris-HCl, $\mathrm{pH} 7.4 ; 25 \mathrm{mM} \mathrm{KCl}$; and $1 \%$ Triton X-100) containing a complete protease inhibitor cocktail (Roche Diagnostics, Tokyo, Japan). Homogenates were centrifuged at $12,000 \times g$ for $10 \mathrm{~min}$ at $4{ }^{\circ} \mathrm{C}$, and aliquots of supernatants were used for subsequent analyses. The protein concentrations of the aliquots were measured using a Coomassie Protein Assay Kit (Thermo Fisher Scientific K.K., Yokohama, Japan). The absorbance was read at $595 \mathrm{~nm}$ using a microplate reader (Model 680; Bio-Rad Laboratories, Inc., CA, USA) to determine the total protein concentrations. The aliquots were diluted with extraction buffer, and EzApply (ATTO, Tokyo, Japan) was added to the diluted aliquots. The final protein concentration of the samples for western blot analysis was adjusted to $2 \mu \mathrm{g} / \mu \mathrm{l}$. For the detection of PGC- $1 \alpha$ and HSP25, the samples were boiled for $5 \mathrm{~min}$; in contrast, for the Total OXPHOS Rodent WB antibody (Abcam, Cambridge, UK), they were not boiled. The samples were applied to a $12.5 \%$ polyacrylamide gel (ATTO), and electrophoresis was performed at a constant 
current of $20 \mathrm{~mA} / \mathrm{gel}$ for $60 \mathrm{~min}$. The separated proteins were then transferred to PVDF membranes (ATTO) at a constant current of $2 \mathrm{~mA} / \mathrm{cm}^{2}$ for $60 \mathrm{~min}$ via the semi-dry blotting method. The membranes were stained with Ponceau-S staining solution (Beacle, Inc., Kyoto, Japan) for $5 \mathrm{~min}$ and the background was destained by $1 \%$ acetic acid for $2 \mathrm{~min}$. The membranes were scanned and destained with $0.1 \mathrm{M} \mathrm{NaOH}$ for $20 \mathrm{~s}$. The membranes were blocked with EzBlock Chemi (ATTO) for $1 \mathrm{~h}$ at room temperature and subsequently incubated overnight at $4{ }^{\circ} \mathrm{C}$ with the following primary antibodies: anti PGC- $1 \alpha$ (1:5000; Santa Cruz Biotechnology, Santa Cruz, USA), Total OXPHOS Rodent WB antibody Cocktail (1:10,000; Abcam), and anti-HSP25 (1:5000; Stressgen Biotechnologies, Victoria, Canada). Following incubation, the membranes were washed 3 times for $10 \mathrm{~min} /$ wash in EzWash (ATTO) containing 0.1\% Tween 20 (TTBS) and reacted for $1 \mathrm{~h}$ at room temperature with the following secondary horse radish peroxidase-conjugated antibodies: anti-mouse IgG (1:20,000; Nacalai Tesque, Kyoto, Japan) for Total OXPHOS, and anti-rabbit IgG (1:25,000; Nacalai Tesque) for PGC- $1 \alpha$ and HSP25. Following this incubation, the membranes were washed 3 times for $10 \mathrm{~min} /$ wash in TTBS and reacted with ECL Prime Western Blot Detection Reagent (GE Healthcare UK Ltd., Buckinghamshire, England) for $5 \mathrm{~min}$ at room temperature, and the protein bands were obtained using LumiCube (Liponics, Inc., Tokyo, Japan). The protein bands were analyzed using JustTLC software (Sweday, Sondra Sandby, Sweden). The 45-kDa bands from the Ponceau-stained membranes were used as protein loading controls [16]. The data were normalized to the Con value.

\section{Histochemical stain}

Muscles were sliced into $10-\mu \mathrm{m}$-thick cross-sections using a cryostat at $-20{ }^{\circ} \mathrm{C}$. Sections were dried at room temperature for $30 \mathrm{~min}$, fixed in 10\% formalin solution for $15 \mathrm{~min}$ at $4{ }^{\circ} \mathrm{C}$, and then stained with hematoxylin-eosin (H\&E). Stained sections were observed under a microscope, and images were obtained using a digital camera.

\section{Statistical analysis}

Comparisons among the three groups were performed using one-way analysis of covariance (ANOVA). Fisher's least significant difference post hoc test was performed to determine significant differences between the groups, based on one-way ANOVA. Statistical analyses were performed using Ekuseru-Toukei 2008 (Social Survey Research Information Co., Ltd., Tokyo, Japan), and statistical significance was set at $p<0.05$.

\section{Results}

\section{Body weight}

Body weight data are shown in Table 1 . There were no significant differences among the three groups in both the initial and final body weights of the mice.

\section{Cholesterol concentration}

To verify the effect of statin on serum cholesterol concentrations, total cholesterol and low-density lipoprotein (LDL) cholesterol levels were measured. The cholesterol concentrations detected in the different mouse treatment groups are shown in Table 1. Total cholesterol levels decreased in the Statin and Statin + Ex groups compared to those in the Con group ( $p=0.011$ and $p=0.029$, respectively). LDL cholesterol levels also decreased in the Statin and Statin + Ex groups compared to those in the Con group ( $p=0.0063$ and $p=0.0025$, respectively).

\section{PGC-1a content}

To verify mitochondrial adaptation by exercise during atorvastatin administration, the level of PGC- $1 \alpha$, which regulates oxidative phosphorylation [17], was measured by western blotting. The PGC- $1 \alpha$ content in the different groups is shown in Fig. 1. The PGC- $1 \alpha$ content was significantly higher in the Statin + Ex group than in the Statin group ( $210 \%$ of that in the Statin group, $p=0.021$ ). However, there was no significant difference in PGC- $1 \alpha$ content between the Con and Statin groups $(p=0.44)$. These results indicate that endurance exercise increases PGC- $1 \alpha$ content during atorvastatin administration.

Table 1 Body weights and cholesterol concentrations in the three groups

\begin{tabular}{lllc}
\hline & Con & Statin & Statin + Ex \\
\hline Initial body weight $(\mathrm{g})$ & $22.0 \pm 0.5$ & $22.4 \pm 1.0$ & $22.8 \pm 0.6$ \\
Final body weight $(\mathrm{g})$ & $26.6 \pm 0.6$ & $26.4 \pm 0.9$ & $27.3 \pm 0.6$ \\
Total cholesterol $(\mathrm{mg} / \mathrm{dl})$ & $93.8 \pm 3.8$ & $78.0 \pm 4.1^{*}$ & $80.5 \pm 3.9^{*}$ \\
LDL cholesterol $(\mathrm{mg} / \mathrm{dl})$ & $10.2 \pm 1.7$ & $5.5 \pm 0.3^{* *}$ & $4.8 \pm 0.3^{* *}$ \\
\hline
\end{tabular}

Values are presented as the mean \pm SE. $n=6$ per group

$L D L$ low-density lipoprotein, Con control, Statin $+E x$ statin and exercise

$* p<0.05$ and $* * p<0.01$ vs the Con group 


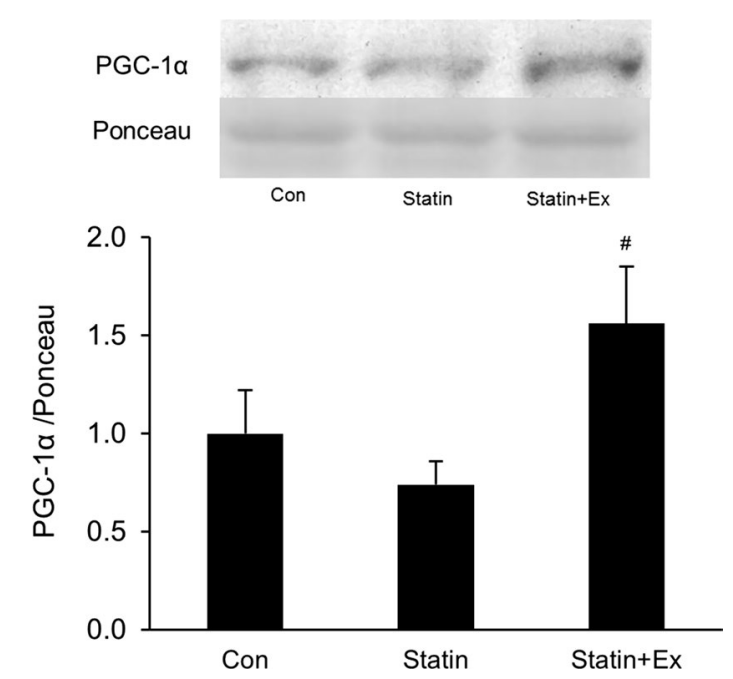

Fig. 1 Expression levels of peroxisome proliferator-activated receptor gamma coactivator 1-alpha (PGC-1 $\alpha)$ in the quadriceps femoris. The expression pattern and protein concentrations of PGC- $1 \alpha$ in the quadriceps femoris in the three groups are shown. Values are expressed as the mean \pm SE. $n=6$ per group. ${ }^{\#} p<0.05$ vs the Statin group. Con control group, Statin $+E x$ statin and exercise group

\section{COX I-V content}

To verify mitochondrial adaptation in response to exercise during atorvastatin administration, the level of COX I-V, which is associated with ATP production in mitochondria, was measured by western blotting. The COX I-V content in the different groups is shown in Fig. 2. The content of COX I, II, III, and IV was significantly higher in the Statin + Ex group than in the Statin group (219\% of Statin; $p=0.032$, $169 \%$ of Statin; $p=0.019,200 \%$ of Statin; $p=0.013$ and $265 \%$ of Statin; $p=0.0036$, respectively). However, there was no significant difference in the content of COX I, II, III, and IV between the Con and Statin groups ( $p=0.28,0.86$, 0.33 , and 0.44 , respectively).

The COX V content was significantly higher in the Statin + Ex group than in the Statin group (207\% of Statin, $p=0.0003$ ). Although there was no significant difference between the Con and Statin groups, COX V levels tended to be lower in the Statin group than in the Con group $(p=0.058)$. These results indicate that endurance exercise increases COX I-V content during atorvastatin administration.
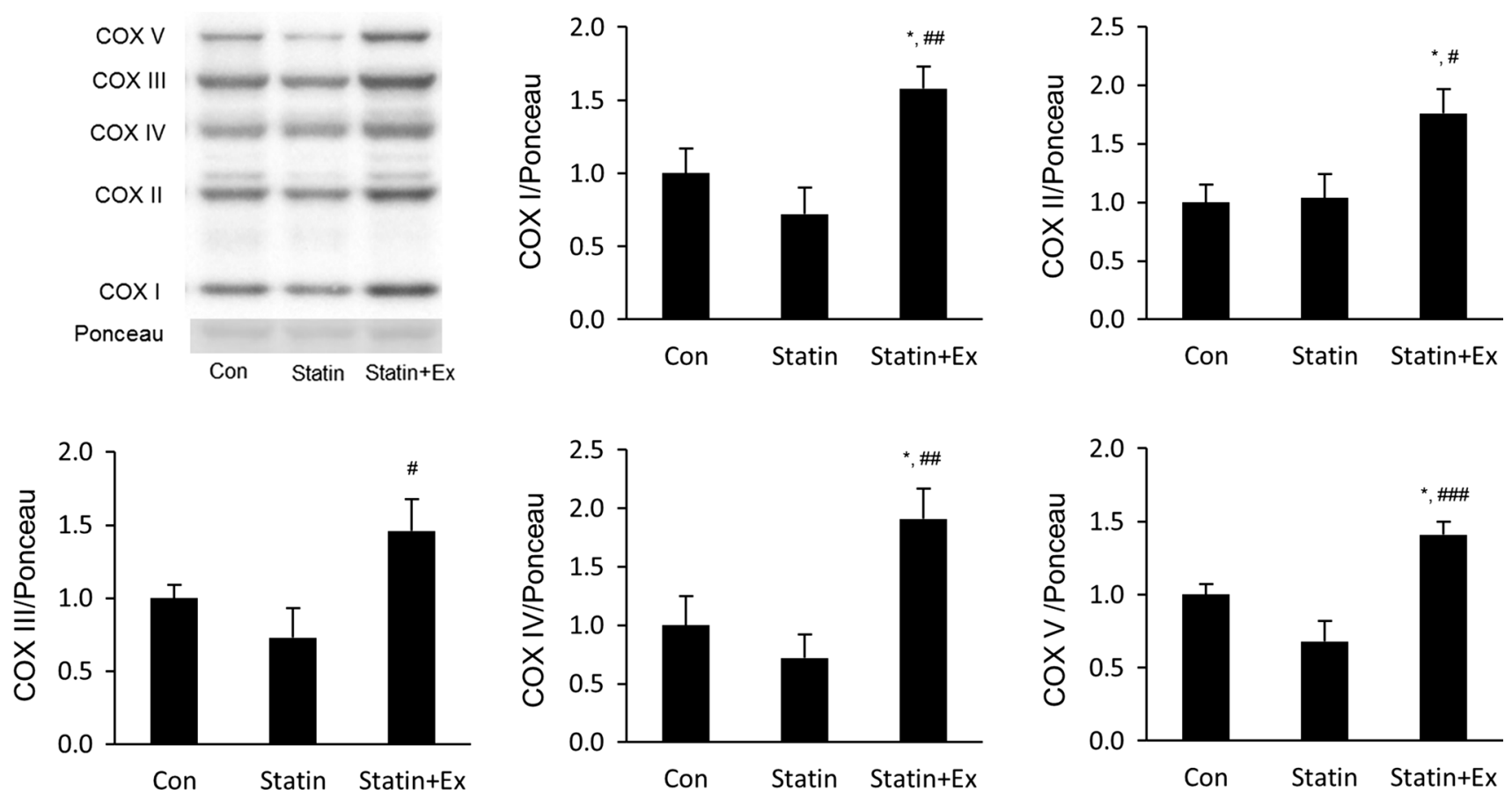

Fig. 2 Expression levels of respiratory chain complex (COX) I-V in the quadriceps femoris. The expression pattern and protein concentrations of COX I-V in the quadriceps femoris in the three groups are presented. Values are expressed as the mean \pm SE. $n=6$ per group.
${ }^{*} p<0.05$ vs the Con group; ${ }^{\#} p<0.05,{ }^{\# \#} p<0.01$ and ${ }^{\# \# \#} p<0.001$ vs the Stain group. Con control group, Statin $+E x$ statin and exercise group 


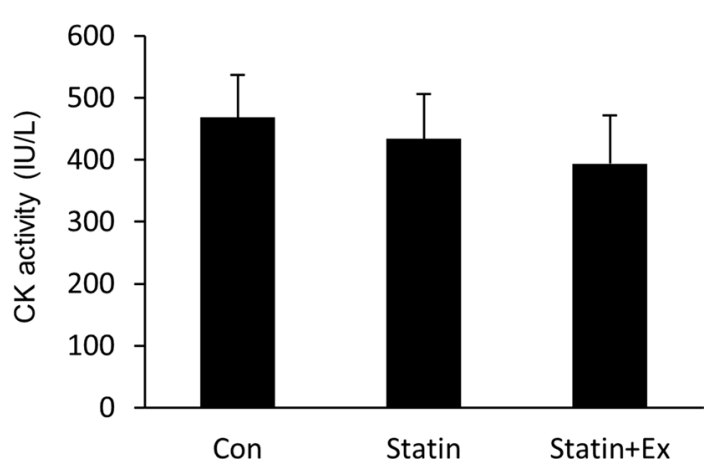

Fig. 3 Creatine kinase (CK) level in serum. Values are expressed as the mean \pm SE. $n=6$ per group. Con control group, Statin $+E x$ statin and exercise group

\section{Serum CK levels}

To verify muscle injury, serum levels of $\mathrm{CK}$, which is a marker of muscle injury, were measured. The CK level is shown in Fig. 3. There was no significant difference in $\mathrm{CK}$ level among the three groups (one-way ANOVA, $p=0.82$ ). The results indicate that endurance exercise did not induce muscle injury during atorvastatin administration.

\section{H\&E stain}

To verify muscle fiber injury, quadriceps femoris sections stained by H\&E were observed by microscopy (Fig. 4). In images of the muscle stained by H\&E, necrotic and regenerative muscle fibers were not observed in any of the groups.

\section{HSP25 content}

The level of HSP25, which is increased by exercise and is expected to protect muscle from injury, was measured by western blotting. The HSP25 levels in the different treatment

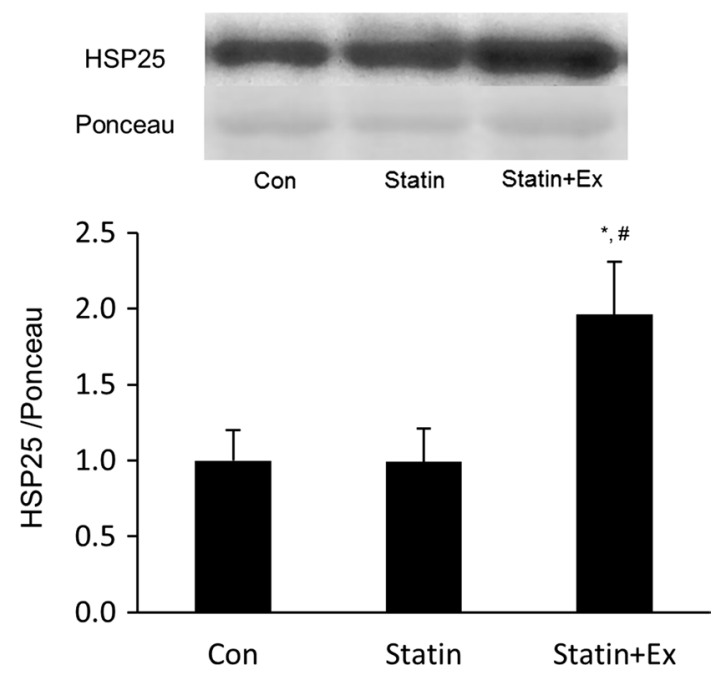

Fig. 5 Expression levels of heat shock protein 25 (HSP25) in the quadriceps femoris. The expression pattern and protein concentrations of HSP25 in the quadriceps femoris in the three groups are shown. Values are expressed as the mean \pm SE. $n=6$ per group. ${ }^{*} p<0.05$ vs the Con group; ${ }^{*} p<0.05$ vs the Statin group. Con control, Statin + Ex statin and exercise group

groups are shown in Fig. 5. HSP25 content was significantly higher in the Statin + Ex group than in the Statin group (197\% of Statin, $p=0.021$ ). These results indicate that endurance exercise increases HSP25 content during atorvastatin administration.

\section{Discussion}

The purpose of this study was to investigate whether endurance exercise induces adaptations in mitochondrialassociated proteins and muscle injury during atorvastatin administration. The major findings in this study were as follows: (1) endurance exercise increased the level of

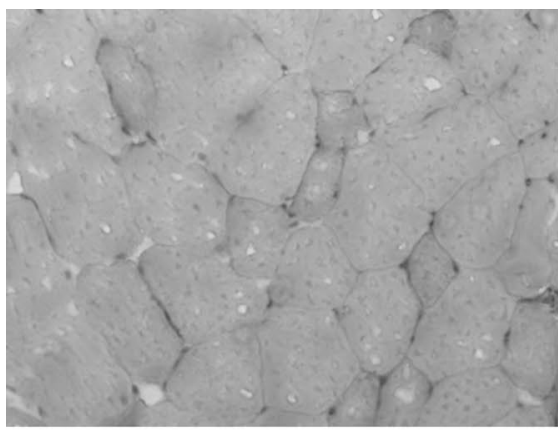

Con

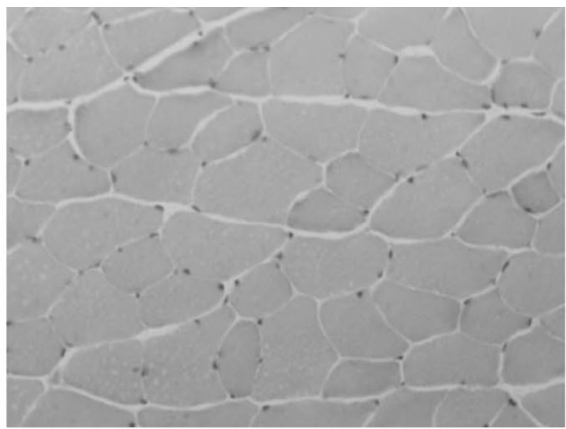

Statin

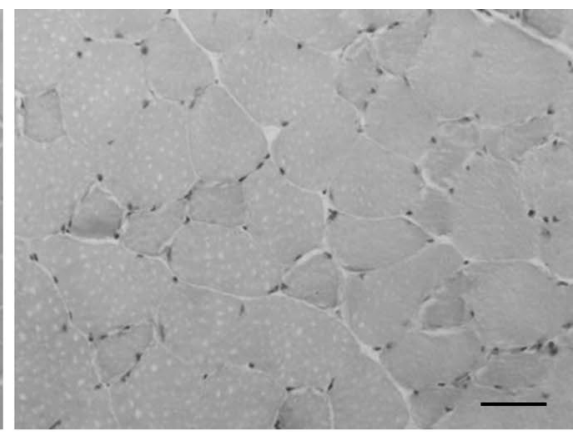

Statin+Ex

Fig. 4 Hematoxylin and eosin (H\&E)-stained cross-sections of the quadriceps femoris. Scale bar $=50 \mu \mathrm{m}$. Con control, Statin + Ex statin and exercise group 
mitochondria-associated proteins such as PGC- $1 \alpha$ and COX I-V during atorvastatin administration; and (2) endurance exercise did not induce skeletal muscle injury during atorvastatin administration. These findings suggest that endurance exercise has adaptive effects on mitochondria-associated proteins in skeletal muscle during atorvastatin administration, without causing muscle injury.

Endurance exercise increased the protein levels of PGC- $1 \alpha$ and COX I-V in the quadriceps femoris muscles of mice during atorvastatin administration, suggesting that endurance exercise has adaptive effects on mitochondria in skeletal muscle during statin administration. Consistent with our results, Southern et al. reported that mitochondria in the skeletal muscles of mice adapted to wheel running during simvastatin treatment [6]. PGC- $1 \alpha$ is known to regulate oxidative phosphorylation genes in skeletal muscle [18]. Overexpression of PGC- $1 \alpha$ has been reported to upregulate mRNA of COX II and COX IV in C2C12 myoblasts and myotubes [19] and in skeletal muscles of mice [20]. Moreover, Wu et al. also reported that COX IV protein level was increased in $\mathrm{C} 2 \mathrm{C} 12$ myoblasts and myotubes overexpressing PGC-1 $\alpha$ [19]. In this study, exercise during atorvastatin administration increased the protein level of PGC- $1 \alpha$, thereby upregulating COX I-V proteins.

In contrast, simvastatin has been reported to impair exercise training adaptation in humans [7], which contradicts our findings. These differences may be due to variations in the dose and type of statin administered. Although the mechanisms underlying statin-induced mitochondrial dysfunction are unclear, the decrease in the levels of ubiquinone, which is produced by the mevalonate pathway, is thought to be one of the causes of mitochondrial dysfunction. Previous studies have reported that a high dose of simvastatin $(80 \mathrm{mg} / \mathrm{kg})$ decreased ubiquinone levels [21]; however, a low dose of simvastatin $(20 \mathrm{mg} / \mathrm{kg})$ did not reduce ubiquinone levels in human skeletal muscle [22]. In addition, Schick et al. [21] reported that simvastatin $(80 \mathrm{mg} / \mathrm{kg})$ decreased ubiquinone levels, whereas atorvastatin $(40 \mathrm{mg} / \mathrm{kg})$ did not have such an effect on human skeletal muscle. These findings suggest that whether or not mitochondrial dysfunction occurs depends on the dose and type of statin administered. In the present study, PGC- $1 \alpha$ and COX I-V levels did not decrease in the mouse quadriceps femoris upon atorvastatin administration. These results suggest that the dose of atorvastatin used in our study did not induce mitochondrial dysfunction in skeletal muscle. However, cases in which a higher dose of atorvastatin or other statins were used, which can induce mitochondrial dysfunction, were associated with impaired mitochondrial adaptation caused by endurance exercise.

Total and LDL cholesterol levels in serum were decreased by atorvastatin administration $(10 \mathrm{mg} / \mathrm{kg} / \mathrm{day})$. It has been reported that serum cholesterol level is not related to treadmill exercise performance in humans [23].
Southern et al. reported that simvastatin administration did not affect mice running capacity [6]. These reports indicate that serum cholesterol level does not affect exercise performance. Therefore, we also think that, in this study, the low cholesterol level induced by atorvastatin administration did not affect endurance exercise capacity and mitochondria.

In our study, we found that endurance exercise did not induce muscle injury during atorvastatin treatment, given that the serum CK level did not increase and necrotic and regenerating muscle fibers, that have central nuclei, were not observed in H\&E-stained tissues. Moreover, the absence of apparent difference in body mass either at the initial or final stage of the exercise protocol may indicate a maintained muscle mass without injury during the administration of atorvastatin. It has been suggested that exercise may exacerbate muscle injury during statin administration [24]; however, we did not observe such an effect in our study. Two reasons may underlie this difference. First, the increase in the level of HSP25 induced by endurance exercise might protect skeletal muscles from mechanical stress during atorvastatin administration. Exercise increases HSP levels in skeletal muscle [12], and the produced HSPs are expected to protect muscle tissue from injury [13]. A higher level of HSP25 was also observed in the quadriceps femoris in the Statin + Ex group compared to that in the Statin group, which is consistent with the results of a previous study [12]. Therefore, endurance exercise increased the level of HSP25, preventing muscle injury from mechanical stress during atorvastatin administration. Second, the intensity of exercise was not strong enough to induce muscle injury during atorvastatin administration. Athletes with statin treatment have a higher prevalence of myopathy (75\%) than statin users who perform exercise (25\%) [24]. Athletes are expected to perform high-intensity exercise, resulting in a high prevalence of myopathy. Therefore, it is possible that the exercise intensity was not high enough to induce muscle injury during atorvastatin treatment.

There are some limitations to this study. First, the higher statin dose and type of statin used have the potential to impair mitochondrial adaptation and affect the endurance exercise-induced muscle injury. Second, a higher intensity of exercise than that used in our study may induce muscle injury during statin administration. However, our study, along with several other reports, suggests that endurance exercise can have adaptive effects on mitochondria-associated proteins in skeletal muscle during atorvastatin administration, without causing muscle injury.

Endurance exercise upregulated protein levels of PGC- $1 \alpha$ and COX I-V in skeletal muscle of mice during atorvastatin administration. In addition, there was no evidence of skeletal muscle injury, such as increased CK activity and necrotic or regenerating muscle fibers. Taken together, our findings 
indicate that endurance exercise induced mitochondrial adaptation in skeletal muscle without muscle injury.

Acknowledgements We would like to thank Editage (www.editage.jp) for English language editing.

Author contributions $\mathrm{KN}$ contributed to experimental design, data collection, analysis, and manuscript preparation. YO contributed to experimental design and data collection. KI contributed to experimental design and manuscript preparation. MS, SU, and JA contributed to manuscript preparation.

\section{Compliance with ethical standards}

Conflict of interest The authors declare that they have no conflict of interest.

Ethical approval All applicable international, national, and/or institutional guidelines for the care and use of animals were followed. All procedures performed in studies involving animals were in accordance with the ethical standards of the institution at which the studies were conducted.

\section{References}

1. Endo A (1992) The discovery and development of HMG-CoA reductase inhibitors. J Lipid Res 33:1569-1582

2. Mills EJ, Rachlis B, Wu P, Devereaux PJ, Arora P, Perri D (2008) Primary prevention of cardiovascular mortality and events with statin treatments. A network meta-analysis involving more than 65,000 patients. J Am Coll Cardiol 52:1769-1781

3. Shiroma EJ, Lee I-M (2010) Physical activity and cardiovascular health: lessons learned from epidemiological studies across age, gender, and race/ethnicity. Circulation 122:743-752

4. Befroy DE, Petersen KF, Dufour S, Mason GF, Rothman DL, Shulman GI (2008) Increased substrate oxidation and mitochondrial uncoupling in skeletal muscle of endurance-trained individuals. Proc Natl Acad Sci USA 105:16701-16706

5. Muraki A, Miyashita K, Mitsuishi M, Tamaki M, Tanaka K, Itoh H (2012) Coenzyme Q10 reverses mitochondrial dysfunction in atorvastatin-treated mice and increases exercise endurance. J Appl Physiol 113:479-486

6. Southern WM, Nichenko AS, Shill DD, Spencer CC, Jenkins NT, McCully KK, Call JA (2017) Skeletal muscle metabolic adaptations to endurance exercise training are attainable in mice with simvastatin treatment. PLoS One 12:1-17

7. Mikus CR, Boyle LJ, Borengasser SJ, Oberlin DJ, Naples SP, Fletcher J, Meers GM, Ruebel M, Laughlin MH, Dellsperger KC, Fadel PJ, Thyfault JP (2013) Simvastatin impairs exercise training adaptations. J Am Coll Cardiol 62:709-714

8. Seachrist JL, Loi CM, Evans MG, Evans MG, Criswell KA, Rothwell CE (2005) Roles of exercise and pharmacokinetics in cerivastatin-induced skeletal muscle toxicity. Toxicol Sci 88:551-561
9. Thompson PD, Zmuda JM, Domalik LJ, Zimet RJ, Staggers J, Guyton JR (1997) Lovastatin increases exercise-induced skeletal muscle injury. Metabolism 46:1206-1210

10. Armstrong RB, Ogilvie RW, Schwane JA (1983) Eccentric exercise-induced injury to rat skeletal muscle. J Appl Physiol 54:80-93

11. Nakayama A, Aoi W, Takami M, Hirano N, Ogaya Y, Wada S, Higashi A (2018) Effect of downhill walking on next-day muscle damage and glucose metabolism in healthy young subjects. $\mathrm{J}$ Physiol Sci 20:1-8

12. Meador BM, Huey KA (2011) Statin-associated changes in skeletal muscle function and stress response after novel or accustomed exercise. Muscle Nerve 44:882-889

13. Koh TJ (2002) Do small heat shock proteins protect skeletal muscle from injury? Exerc Sport Sci Rev 30:117-121

14. Bouitbir J, Charles A-L, Rasseneur L, Dufour S, Piquard F, Geny B, Zoll J (2011) Atorvastatin treatment reduces exercise capacities in rats: involvement of mitochondrial impairments and oxidative stress. J Appl Physiol 111:1477-1483

15. Ferreira JCB, Rolim NPL, Bartholomeu JB, Gobatto CA, Kokubun E, Brum PC (2007) Maximal lactate steady state in running mice: effect of exercise training. Clin Exp Pharmacol Physiol 34:760-765

16. Perry RA, Brown LA, Lee DE, Brown JL, Baum JI, Greene NP, Washington TA (2016) The Akt/mTOR pathway: data comparing young and aged mice with leucine supplementation at the onset of skeletal muscle regeneration. Data Brief 8:1426-1432

17. Lira V, Benton C, Yan Z, Bonen A (2010) PGC-1alpha regulation by exercise training and its influences on muscle function and insulin sensitivity. Am J Physiol 299:E145-E161

18. Handschin C, Spiegelman BM (2006) Peroxisome proliferatoractivated receptor $\gamma$ coactivator 1 coactivators, energy homeostasis, and metabolism. Endocr Rev 27:728-735

19. Wu Z, Puigserver P, Andersson U, Zhang C, Adelmant G, Mootha V, Troy A, Cinti S, Lowell B, Scarpulla RC, Spiegelman BM (1999) Mechanisms controlling mitochondrial biogenesis and respiration through the thermogenic coactivator PGC-1. Cell 98:115-124

20. Miura S, Kai Y, Ono M, Ezaki O, Ezaki O (2003) Overexpression of peroxisome proliferator-activated receptor $\gamma$ coactivator- $1 \alpha$ down-regulates GLUT4 mRNA in skeletal muscles. J Biol Chem 278:31385-31390

21. Schick BA, Laaksonen R, Frohlich JJ, Päivä H, Lehtimäki T, Humphries KH, Côté HC (2007) Decreased skeletal muscle mitochondrial DNA in patients treated with high-dose simvastatin. Clin Pharmacol Ther 81:650-653

22. Laaksonen R, Jokelainen K, Laakso J, Sahi T, Harkonen M, Tikkanen MJ, Himberg JJ (1996) The effect of simvastatin treatment on natural antioxidants in low-density lipoproteins and highenergy phosphates and ubiquinone in skeletal muscle. Am J Cardiol 77:851-854

23. Leon AS, Jacobs DR, DeBacker G, Taylor HL (1981) Relationship of physical characteristics and life habits to treadmill exercise capacity. Am J Epidemiol 113:653-660

24. Meador BM, Huey KA (2010) Statin-associated myopathy and its exacerbation with exercise. Muscle Nerve 42:469-479 